\title{
Utility of Diffusion Tensor Imaging in Evaluation of the Peritumoral Region in Patients with Primary and Metastatic Brain Tumors
}

\author{
E.J. Sternberg, M.L. Lipton, and J. Burns
}

\begin{abstract}
SUMMARY: In the brain, diffusion tensor imaging is a useful tool for defining white matter anatomy, planning a surgical approach to space-occupying lesions, and characterizing tumors, including distinguishing primary tumors from metastases. Recent studies have attempted, with varying success, to use DTI to define the extent of tumor microinfiltration beyond the apparent borders on T2-weighted imaging. In the present review, we discuss the current state of research on the utility of DTI for evaluating the peritumoral region of brain tumors.
\end{abstract}

ABBREVIATIONS: $\mathrm{FA}=$ fractional anisotropy; $\mathrm{MD}=$ mean diffusivity; $\mathrm{TI}=$ tumor infiltration index

M alignant brain tumors such as glioblastoma multiforme have an infiltrative pattern of growth, often invading the surrounding tissue. ${ }^{1,2}$ Actual tumor margins can extend microscopically for several centimeters past the radiographically detected margin of disease. ${ }^{3}$ Furthermore, infiltrative brain tumors are surrounded by extensive areas of edema, as detected on T2weighted imaging. The transition between the tumor edge and peritumoral edema is ambiguous. In metastatic tumors, this peritumoral edema is thought to consist of pure water, which can obscure tumor borders. However, the peritumoral edema of infiltrative tumors such as glioblastoma multiforme has often been shown to contain tumor cells that have spread into the edematous tissue. ${ }^{4}$ Conventional imaging techniques such as MR imaging and CT are not able to detect this minute cellular infiltration, and studies show that tumor cells have been demonstrated beyond tumor borders as defined by these modalities. ${ }^{5-8}$ This limitation of conventional CT and MR imaging presents a serious challenge for treatment planning. In successful surgical resection or biopsy with adjuvant therapy, the tumor must be excised to the greatest extent possible while minimizing injury to nearby healthy tissue.

Received May 9, 2013; accepted after revision June 12.

From Tufts University School of Medicine (E.J.S.), Boston, Massachusetts; the Gruss Magnetic Resonance Research Center and Departments of Radiology, Psychiatry, and Behavioral Sciences and the Dominick P. Purpura Department of Neuroscience (E.J.S., M.L.L.), Albert Einstein College of Medicine, Bronx, New York; Department of Radiology (M.L.L., J.B.), Montefiore Medical Center, Bronx, New York.

The authors declare that there are no conflicts of interest and no competing financial interests.

Please address correspondence to Judah Burns, MD, Division of Neuroradiology, Department of Radiology, Montefiore Medical Center, Albert Einstein College of Medicine 111 E 210th St, New York, NY 10467; e-mail: jburns@montefiore.org

http://dx.doi.org/10.3174/ajnr.A3702
Standard imaging techniques tend to underestimate the extent of tumor involvement, a problem that can lead to suboptimal treatment and a worse prognosis. ${ }^{9}$

Studies of animal models provide insight into the mechanisms of change in DTI metrics and imply that DTI may have a role in more precisely defining the extent of tumor cell infiltration. Among other applications, DTI has been successfully used in animals for mapping white matter tracts, ${ }^{10}$ for characterizing the directionality of diffusion within tumors, ${ }^{11}$ and as a marker of pathologic states such as demyelination. ${ }^{12}$ Several animal studies have also used DTI to detect tumor microinfiltration into surrounding tissue, by using histologic analysis as the criterion standard. ${ }^{13,14}$ For example, Lope-Piedrafita et al (2008) $)^{15}$ implanted glioma cells (C6) into the brains of 6 adult rats and, by using DTI metrics, showed that fractional anisotropy (FA) values in the tissue contralateral to the tumor were relatively high and did not change significantly with time, while the FA values adjacent to the tumor, though they were initially similar to those of the contralateral side, tended to decrease as the tumor grew. This reduction in fractional anisotropy became significant by day 6 of growth, suggesting that as the tumor grew, cancer cells infiltrated the tissue surrounding the tumor. The introduction of these more randomly distributed cells among white matter axons caused a reduction in the uniformity of water diffusion within tissue, leading to lower FA values of the peritumoral white matter on DTI.

In a similar study, Kim et al $(2008)^{16}$ used DTI to monitor the growth of rapid-growing F98 and the slower growing 9L tumor models in rats. In evaluating the peritumoral regions, they found that due to tissue compression from rapid growth, the coefficient of planar anisotropy was higher surrounding 


\begin{tabular}{|c|c|c|c|c|c|c|}
\hline Article & No. & Design & Measures & Tumor types & $\begin{array}{l}\text { Criterion } \\
\text { Standard }\end{array}$ & Conclusion $^{a}$ \\
\hline Deng et al, $2010^{27}$ & 20 & Prospective cohort study & FA, ADC & $\triangle$ Primary $\square$ Metastasis $\square$ Extra-axial & Tissue & Yes \\
\hline Wang et al, $2009^{28}$ & 49 & Retrospective analysis & $F A, p, q, L$ & $凶$ Primary $\square$ Metastasis $\square$ Extra-axial & None & Yes \\
\hline Price et al, $2006^{29}$ & 20 & Prospective cohort study & $F A, p, q$ & 冈Primary $₫$ Metastasis $\square$ Extra-axial & Tissue & Yes \\
\hline Sinha et al, $2002^{13}$ & 9 & Prospective cohort study & FA, MD & $凶$ Primary $\square$ Metastasis $\square$ Extra-axial & None & No \\
\hline Provenzale et al, $2004^{26}$ & 17 & Retrospective analysis & FA, ADC & $凶$ Primary $\square$ Metastasis $\otimes$ Extra-axial & None & Yes \\
\hline Lu et al, $2003^{20}$ & 24 & Prospective cohort study & $\mathrm{FA}, \mathrm{MD}$ & $凶$ Primary $\bigotimes$ Metastasis $\square$ Extra-axial & None & Yes \\
\hline Lu et al, $2004^{24}$ & 40 & Retrospective analysis & FA, MD, TII & 凶Primary $\bigotimes$ Metastasis $\bowtie$ Extra-axial & None & Yes \\
\hline Price et al, $2003^{23}$ & 20 & Prospective cohort study & RAI & $凶$ Primary $\bowtie$ Metastasis $\square$ Extra-axial & None & Yes \\
\hline Sundgren et al, $2006^{21}$ & 28 & Prospective cohort study & FA, ADC & $凶$ Primary $\otimes$ Metastasis $\square$ Extra-axial & Follow-up & Yes \\
\hline van Westen et al, $2006^{14}$ & 30 & Prospective cohort study & FA, ADC & 冈Primary $\bigotimes$ Metastasis $\bigotimes$ Extra-axial & Tissue, cytology & No \\
\hline Kinoshita et al, $2010^{30}$ & 14 & Retrospective analysis & FA, ADC, TII & 凶Primary $\bowtie$ Metastasis $\bowtie$ Extra-axial & None & No \\
\hline Tropine et al, $2004^{25}$ & 12 & Prospective cohort study & $\mathrm{FA}, \mathrm{MD}$ & $凶$ Primary $\square$ Metastasis $\bowtie$ Extra-axial & None & Inconclusive \\
\hline
\end{tabular}

Note:- p indicates pure isotropic vector component; q, pure anisotropic vector component; L, total magnitude of the diffusion tensor; RAl, relative anisotropy index.

a Whether the study data suggest that DTI has utility in determining the extent of brain tumor infiltration.

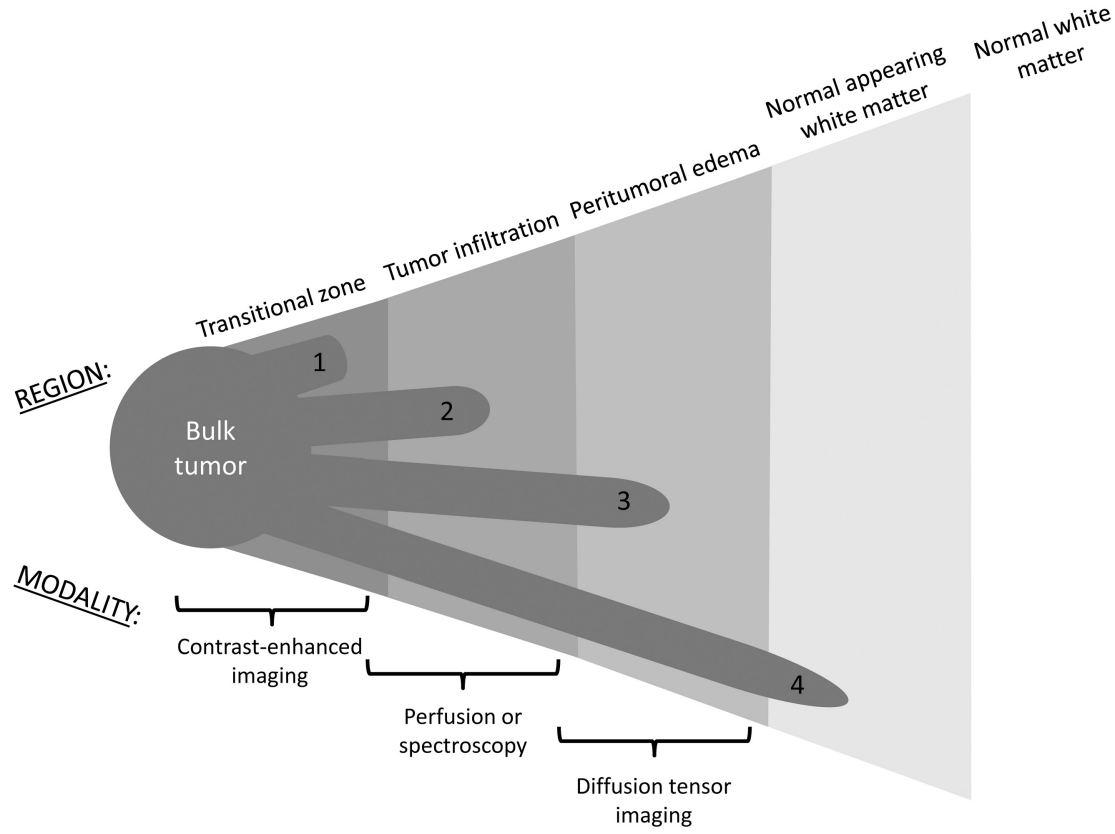

FIG 1. The peritumoral region and corresponding imaging modalities. Tumor cells can extend beyond the bulk tumor into vaguely concentric subdivisions of the peritumoral region, including the transitional zone (1), the region of tumor infiltration (2), the peritumoral edema (3), and even the normal-appearing white matter (4).

both tumors than in the tumor itself. Similarly, also due to mass effect, the tertiary eigenvalue was lower in the 9L peritumoral region compared with the tumor body. In contrast, despite the faster growth of F98 tumors, the tertiary eigenvalue in their peritumoral region was higher than that of the tumor body. It was suggested that this was due to infiltration of tumor cells into the peritumoral tissue, and this hypothesis was supported by histologic analysis of tumor invasiveness. ${ }^{16}$

Findings in animal models suggest that DTI may have a role in human tumor characterization. A number of studies have attempted to use DTI to more precisely delineate the margins of brain tumors in humans and detect changes in the normal-appearing tissue surrounding malignant gliomas that are not detectable on conventional MR imaging. There is good early investigational evidence to support the use of DTI in evaluating the peritumoral region; therefore, focused research in this regard will be needed. The purpose of this review is to highlight the available literature as a basis for further research.

\section{Literature Search Approach and Results}

Using variations of the search term "diffusion tensor imaging brain tumor," we acquired our raw list of articles from the PubMed data base. For completeness, we also looked for relevant articles cited by these publications. Our search yielded 252 articles that discussed DTI. Of those, there were 5 animal studies, 35 case reports, 2 letters, 1 meta-analysis, 137 randomized controlled trials, 21 retrospective studies, and 51 reviews. In terms of content, 33 articles concerned the peritumoral region; 108, white matter tractography and/or surgical approach; 46, bulk tumor characterization; and the rest covered miscellaneous topics. Of the peritumoral articles, 12 directly addressed the question of whether DTI has utility in evaluating tumor infiltration in the peritumoral region (Table). These studies typically used ROIs and an expert rater to evaluate DTI results. A notable limitation in discussing these studies is that they are not consistent in precisely which subdivision of the peritumoral region (Fig 1) they address. FA values vary depending on location, such as whether they are measured in compact or noncompact regions of tissue. This must be taken into account in any DTI analysis so as not to compromise the specificity of study results.

Finally, the tractography results reviewed here are based on deterministic algorithms. Other approaches, such as probabilistic tractography and multishell dMRI, may overcome obstacles to tractography such as crossing-fiber zones and abnormal tissue. While these techniques may improve the delineation of tract-based ROIs in the peritumoral region, this review specifically focuses on assessments of scalar metrics of anisotropy because these comprise the methods that have been reported to date. 


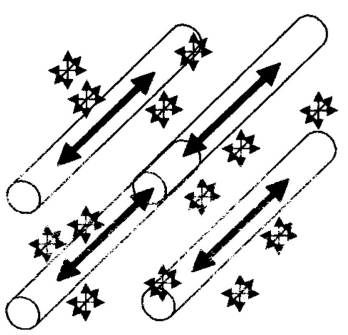

$\uparrow \uparrow$ Water Content Contributing to $\downarrow F A$ A (Metastatic Lesions)

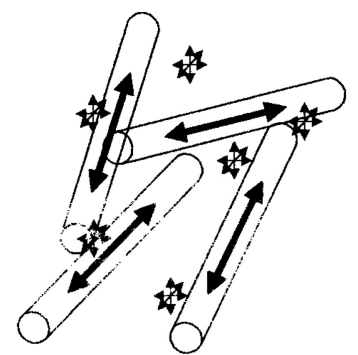

TWater Content and Axonal Disorganization
Contributing to \FA

B (High-Grade Gliomas)

FIG 2. Schematic illustrating the factors behind the comparable change in peritumoral FA. A, Water content contributing to decreased FA in metastatic lesions. $B$, Water content and axonal disorganization contributing to decreased FA in high-grade gliomas. Reprinted from Lu et al. ${ }^{20}$

\section{Distinguishing Primary Brain Tumors from Metastases}

Brain tumors, regardless of whether primary or metastatic, are surrounded by some degree of vasogenic edema, detectable as hyperintensity on T2-weighted images. Diffusion metrics have been shown to be altered within this surrounding edema, compared with internal controls, in both high-grade gliomas and metastatic tumors, reflecting the presence of, at minimum, increased extracellular water. It has been shown further, by using DWI, that the diffusivity of the peritumoral edema varies depending on the characteristics of the tumor it encompasses. High-grade gliomas are associated with edema of high trace value (mean diffusivity [MD], computed by averaging the diagonal elements of the matrix ${ }^{17}$ ), whereas the low-grade gliomas or nonglial tumors are associated with edema of low trace value. Morita et al $(2005)^{18}$ found a similar range of anisotropy in both types of edema, yet the diffusivity was much greater in the edema surrounding highgrade gliomas. They concluded that the "higher diffusivity of water molecules within the area of edema associated with high-grade gliomas is likely to reflect destruction of the extracellular matrix ultrastructure by malignant cell infiltration." 18 This conclusion was supported by a subsequent study by Kimura et $\mathrm{al}^{19}$ by using changes in glutamate levels (as a marker of the integrity of the extracellular matrix) as the central measure. Figure 2 illustrates the factors behind comparable changes in peritumoral FA.

Current research demonstrates that surrounding both primary brain tumors and metastatic tumors, there is an increase in mean diffusivity and a decrease in FA compared with normalappearing white matter, measurements best explained by increased extracellular water. The greater the amount of free water in tissue, the greater the magnitude of diffusion will be (increased $\mathrm{MD}$ ) and the more disorganized the diffusion becomes (decreased FA). ${ }^{19}$ Lu et al $(2003)^{20}$ found that the peritumoral MD of metastatic lesions was greater than that of gliomas, while the peritumoral FA between the 2 tumor types demonstrated no significant difference. This finding implies that the reduced FA surrounding gliomas can be attributed not only to increased extracellular water but also to axonal disorganization caused by infiltration of the tumor beyond its detectable margin.

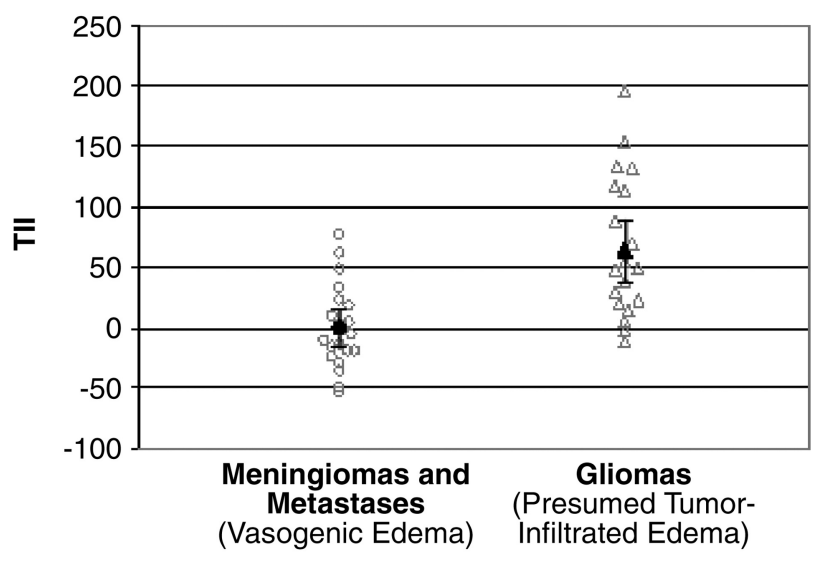

FIG 3. Graph of TII values in meningiomas and metastases compared with TIls in gliomas. The mean TII of meningiomas and metastases is zero, with a $95 \% \mathrm{Cl}$ between -15 and 15 . The mean TII of gliomas is 64 , with a $95 \% \mathrm{Cl}$ between 38 and 90 . Reprinted with permission from Lu S, Ahn D, Johnson G, et al. Diffusion-tensor MR imaging of intracranial neoplasia and associated peritumoral edema: introduction of the tumor infiltration index. Radiology 2004;232:221-28. ${ }^{24}$

\section{Distinguishing Tumor Extension from Edema of the Peritumoral Region}

External support for this notion that reduced FA in peritumoral edema is a marker of tumor infiltration comes from the Sundgren et al (2006) $)^{21}$ study of recurrent and nonrecurrent brain tumors. Tumors that have great infiltrative potential are more likely to recur than tumors lacking such potential. ${ }^{22}$ Sundgren et al measured the FA values in the normal-appearing white matter bordering the peritumoral edema of recurrent and nonrecurrent tumors. These investigators reported significantly lower FA values in the normal tissue surrounding recurrent tumors compared with nonrecurrent tumors, ${ }^{21}$ suggesting the presence of microinfiltration by the recurrent tumors into the peritumoral white matter. This hypothesis could be neatly reconciled with the known correlation between tumors of high infiltrative capacity and the associated recurrence risk. Earlier work has similarly shown that changes in DTI metrics can identify subtle white matter disruption due to occult infiltration by aggressive cancers. ${ }^{23}$

In a second study in 2004, Lu et $\mathrm{al}^{24}$ looked retrospectively at DTI data from 40 patients and attempted to determine whether the MD and FA could be used to distinguish intra-axial from extra-axial lesions, metastatic lesions from gliomas, and high-grade (World Health Organization grades III-IV) from low-grade (World Health Organization grade II) gliomas. However, to better define the extent of tissue infiltration, these investigators used a third metric that they termed the "tumor infiltration index (TII)," which was calculated from DTI data by the following formula:

$$
\mathrm{TII}=(\text { FAexp }-F A o b s) 10^{3},
$$

where FAexp is the expected FA for the corresponding MD, assuming no tumor infiltration (as determined by a linear regression analysis of such data) and FAobs is the currently measured FA. Thus, in order for the results to be consistent with those of the above study, the TII of noninfiltrative tumors should be approximately zero, while the TII of infiltrative tumors should be considerably higher. That is indeed the outcome achieved. Gliomas had a mean TII of 64, while metastases and meningiomas had a mean TII of 0 (Fig 3). 

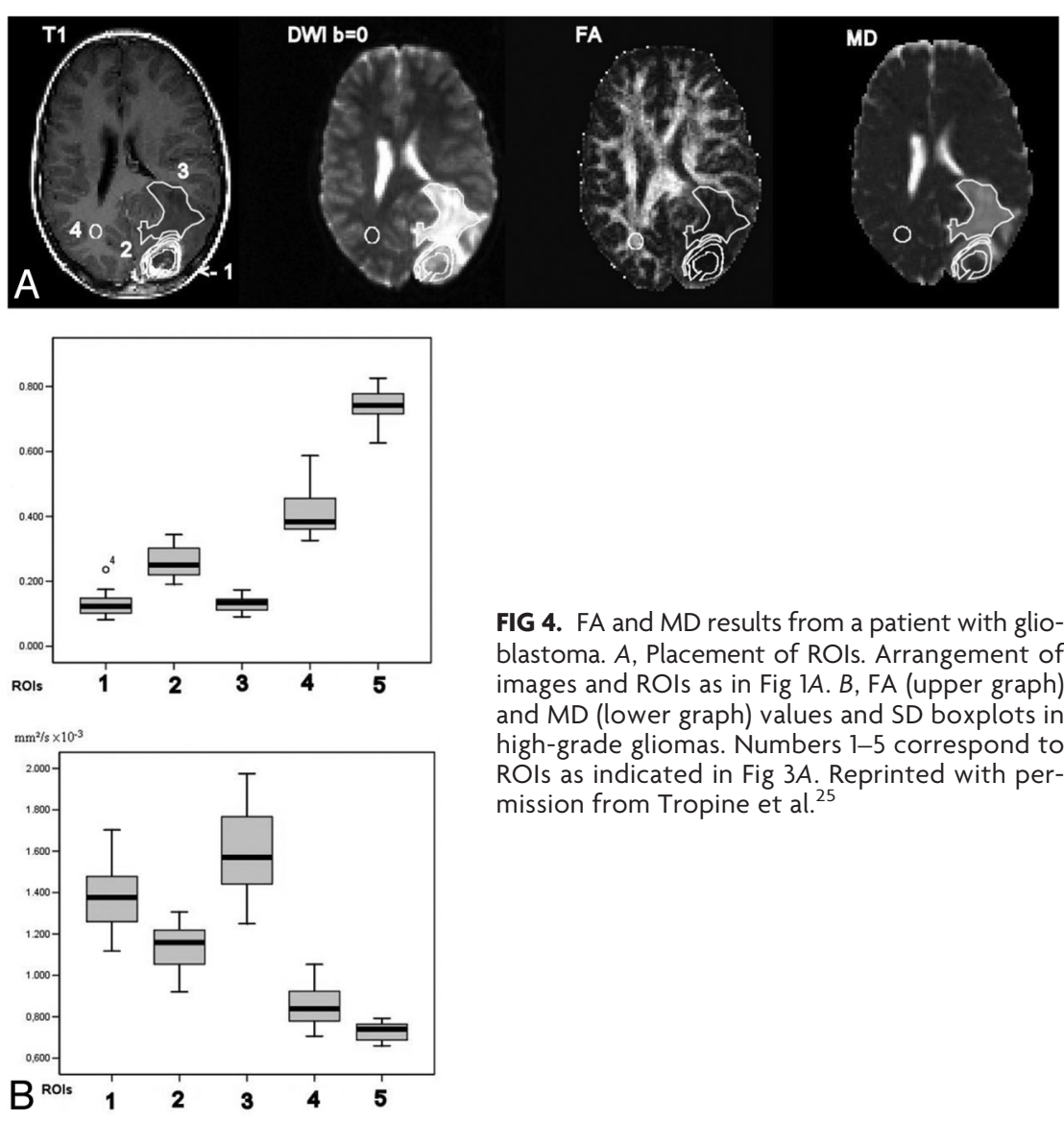

FIG 4. FA and MD results from a patient with glioblastoma. A, Placement of ROls. Arrangement of images and ROIs as in Fig $1 A$. $B$, FA (upper graph) and MD (lower graph) values and SD boxplots in high-grade gliomas. Numbers 1-5 correspond to ROIs as indicated in Fig $3 A$. Reprinted with permission from Tropine et al. ${ }^{25}$

infiltration that cannot be discerned by standard MR imaging techniques.

Recent evidence further supports the notion that DTI, specifically the FA value, is a useful tool in precisely delineating tumor margins. Deng et al $(2010)^{27}$ correlated fractional anisotropy with the distance from visible tumor margins in gliomas and found that the value increases as the distance from the tumor increases. In addition, by obtaining tissue samples from the regions of interest as determined on MR imaging, these investigators observed a negative association between FA values from the peritumoral edema of gliomas and the degree of tumor infiltration (categorized as mild, moderate, severe, or no infiltration). ${ }^{27}$ These results are consistent with past findings that a reduction in FA in the peritumoral region could be used as a quantitative index of tumor cell infiltration.

In addition to $\mathrm{FA}$ and $\mathrm{MD}$, several eigenvalues derived from DTI have been successfully used for studying the extent of brain tumor infiltration. These include the pure isotropic component $(p)$, the pure anisotropic component $(q)$, and the total magnitude of the diffusion tensor $(L)$, where $L=\sqrt{p^{2}+q^{2}} \cdot{ }^{28}$ It has

A number of other studies have emerged that lend support to the notion that DTI metrics might be a useful marker of tumor infiltration into the surrounding tissue. For example, Tropine et al $(2004)^{25}$ found a reduction of FA in the peritumoral, T2 signalenhancing region of glioblastomas compared with meningiomas and concluded that this may be an indicator of tumor cell infiltration, though they questioned the reliability of such a test given the evidence available. DTI results from a patient with glioblastoma are shown in Fig 4.

A similar study by Provenzale et al (2004) ${ }^{26}$ further demonstrated the efficacy of DTI in differentiating normal and tumorinfiltrated peritumoral tissue. The investigators found that mean FA values in peritumoral hyperintense regions were $43 \%$ of the normal white matter value for gliomas and $65 \%$ of the normal white matter value for meningiomas $(P=.05)$, while the mean FA values for normal-appearing white matter neighboring the tumors were $83 \%$ of the normal value for patients with gliomas and $100 \%$ of the normal value for those with meningiomas $(P=$ $.01) .{ }^{26}$ In other words, there was a noticeable but statistically insignificant difference in FA in the hyperintense regions of gliomas and meningiomas. However, the magnitude of the decrease in FA in the normal-appearing, neighboring white matter was significant: The decrement was greater in patients with glioma $(P=.01)$. The fact that DTI analysis reveals a disparity in the FA of peritumoral normal-appearing tissue and that this disparity is greater in the tissue neighboring the gliomas than in neighboring meningiomas perhaps implies that DTI has the potential to detect tumoral been found, for example, that by using the pure diffusion components, one can distinguish gross tumor (reduction of $q>12 \%$ from contralateral region) from tumor infiltration (increase $p>$ $10 \%$ from contralateral region). ${ }^{29}$ These metrics may perhaps strengthen the role of DTI in the analysis of the peritumoral region.

\section{Limitations of DTI in Delineating Tumor Margins}

In contrast to those studies discussed previously, several studies did not find conclusive evidence of the utility of DTI for detecting tumor infiltration. In a study of 9 patients by Sinha et al (2002), ${ }^{13}$ the FA values were significantly different in only 7 of the 9 patients studied and DTI was not found to have any advantage over the more conventional diffusion-weighted MR imaging (the FA differences paralleled the diffusivity differences and did not add any additional insight). With such a small study population, however, this result may not be representative.

A larger study conducted by van Westen et al (2006) ${ }^{14}$ measured the tumor-to-brain ratios of FA and ADC in T2 hyperintense regions surrounding gliomas, meningiomas, and metastases and the adjacent normal-appearing white matter of 30 patients. They found no difference among the 3 tumor types in terms of ADC magnitude, the lesion-to-brain ratios of ADC, the FA magnitude, the lesion-to-brain ratio, or FA within the peritumoral areas with T2 signal changes. Furthermore, they found no difference in the ADC or tumor-to-brain ratios of FA in the adjacent normal-appearing white matter. van Westen et al, therefore, con- 
cluded that the FA was not helpful in identifying tumor-infiltrated edema. In addition, Kinoshita et al $(2010)^{30}$ re-evaluated the utility of the previously discussed tumor infiltration index in 14 patients, comparing DTI with ${ }^{11} \mathrm{C}$-methionine PET, which has been shown to have utility in identifying tumor infiltration. ${ }^{31}$ In glioma cases $(n=8)$, the mean TII ranged from -127 to 96 , with an overall mean of $5.4 \pm 65$. On the other hand, the TII for meningiomas $(n=5)$ and metastatic brain tumors $(n=1)$ ranged from -24 to 44 , with an overall mean of $6.8 \pm 29$. However, these differences were not statistically significant — a finding that questions TII (a measure derived from the FA) as a tool for distinguishing tumor-infiltrated edema from vasogenic edema. However, voxel-by-voxel comparison of TII and ${ }^{11} \mathrm{C}$-methionine PET in the areas of gliomas demonstrating T2-signal hyperintensity showed a positive correlation between the two modalities. This suggests that though TII may perhaps be unable to delineate tumor infiltration within vasogenic edema, it is still useful for identifying tumor cell infiltration into nonedematous regions. ${ }^{32}$ It has been further suggested that ${ }^{11} \mathrm{C}$-methionine PET could be used as an adjunctive study with DTI to help distinguish tumor-infiltrated edema from typical vasogenic edema. ${ }^{33}$

The concern may arise that the integration of results from multiple diffusion MRI studies creates the potential for systematic bias due to differences in data acquisition and analysis methods among studies. This concern is germane when quantitative measures are compared across studies. However, when study parameters (such as hardware, software, data acquisition, and analysis methods) are standardized across all subjects within a study, as in the articles reviewed herein, this concern is minimized, provided that the temporal stability of the diffusion MRI measurement is maintained during the period of data acquisition for the study sample. Because this review was based on the assessment of the findings of other studies but does not compare their quantitative measurements, this concern should be minimal in the context of this review.

\section{CONCLUSIONS}

From the evidence discussed above (Table), we contend that DTI is a sophisticated and useful tool to aid in the delineation of tumor margins and the detection of brain tumor infiltration. The evidence suggests that decreased fractional anisotropy in peritumoral tissue, compared with the corresponding contralateral brain region, is an indicator of tumor invasion past the tumor borders as detected on T2-weighted images. Although the architecture of the tissue can be highly variable and therefore create inconsistent or nonspecific results, the use of adjunctive DTI metrics can improve the quality of findings and facilitate accurate and specific conclusions.

We believe that the technique is promising but that the literature is currently insufficient to establish a definitive clinical role for DTI in the characterization of the peritumoral region. The primary reason for this is that though there have been many studies of the utility of DTI for this novel purpose (Table), few have used a criterion standard such as tissue histopathology to confirm their results. Future studies by using a criterion standard could lay the groundwork for an eventual meta-analysis that could conclusively quantify the overall effectiveness of the technique. In addi- tion, automated translation of DTI metrics to visual anatomic maps may make the process of evaluating the peritumoral region by using DTI more uniform and therefore more clinically relevant. We expect that continued research in this area will further advance the quality and utility of DTI for delineating tumor extent.

\section{REFERENCES}

1. Scherer HJ. The forms of growth in gliomas and their practical significance. Brain 1940;63:1-35

2. Johnson P, Hunt S, Drayer B. Human cerebral gliomas: correlation of postmortem MR imaging and neuropathologic findings. Radiology 1989;170:211-17

3. DeAngelis LM. Brain tumors. N Engl J Med 2001;344:114-23

4. Kelly P, Daumas-Duport C, Kispert D, et al. Imaging-based stereotaxic serial biopsies in untreated intracranial glial neoplasms. J Neurosurg 1987;66:865-74

5. Lilja A, Bergstrom K, Spannare B. Reliability of computed tomography in assessing histopathological features of malignant supratentorial gliomas. J Comput Assist Tomogr 1981;5:625-36

6. Selker RG, Mendelow H, Walker M, et al. Pathological correlation of CT ring in recurrent, previously treated gliomas. Surg Neurol 1982;17:251-54

7. Watanabe $M$, Tanaka R, Takeda N. Magnetic resonance imaging and histopathology of cerebral gliomas. Neuroradiology 1992;34:463-69

8. Burger P, Dubois P, Schold S, et al. Computerized tomographic and pathologic studies of the untreated, quiescent, and recurrent glioblastoma multiforme. J Neurosurg 1983;58:159-69

9. Tovi M. MR imaging in cerebral gliomas analysis of tumour tissue components. Acta Radiol Suppl 1993;384:1-24

10. Asanuma T, Doblas S, Tesiram YA, et al. Diffusion tensor imaging and fiber tractography of $\mathrm{C6}$ rat glioma. J Magn Reson Imaging 2008;28:566-73

11. Zhang J, van Zijl PC, Laterra J, et al. Unique patterns of diffusion directionality in rat brain tumors revealed by high-resolution diffusion tensor MRI. Magn Reson Med 2007;58:454-62

12. Beaulieu C, Allen PS. Determinants of anisotropic water diffusion in nerves. Magn Reson Med 1994;31:394-400

13. Sinha S, Bastin ME, Whittle IR, et al. Diffusion tensor MR imaging of high-grade cerebral gliomas. AJNR Am J Neuroradiol 2002;23:520-27

14. van Westen D, Lätt J, Englund E, et al. Tumor extension in highgrade gliomas assessed with diffusion magnetic resonance imaging: values and lesion-to-brain ratios of apparent diffusion coefficient and fractional anisotropy. Acta Radiol 2006;47:311-19

15. Lope-Piedrafita S, Garcia-Martin ML, Galons JP, et al. Longitudinal diffusion tensor imaging in a rat brain glioma model. NMR in Biomedicine. 2008;21:799-808

16. Kim S, Pickup S, Hsu O, et al. Diffusion tensor MRI in rat models of invasive and well-demarcated brain tumors. NMR in Biomedicine 2008;21:208-16

17. Le BihanD, Mangin JF, Poupon C, et al. Diffusion tensor imaging: concepts and applications. J Magn Reson Imaging 2001;13:534-46

18. Morita K, Matsuzawa H, Fujii Y, et al. Diffusion tensor analysis of peritumoral edema using lambda chart analysis indicative of the heterogeneity of the microstructure within edema. J Neurosurg 2005; 102:336-41

19. Kimura $\mathrm{T}$, Ohkubo $\mathrm{M}$, Igarashi $\mathrm{H}$, et al. Increase in glutamate as a sensitive indicator of extracellular matrix integrity in peritumoral edema: a 3.0-Tesla proton magnetic resonance spectroscopy study. J Neurosurg 2007;106:609-13

20. Lu S, Ahn D, Johnson G, Cha S. Peritumoral diffusion tensor imaging of high grade gliomas and metastatic brain tumors. AJNR Am J Neuroradiol 2003;24:937-41

21. Sundgren PC, Fan X, Weybright $P$, et al. Differentiation of recurrent brain tumor versus radiation injury using diffusion tensor imaging in patients with new contrast-enhancing lesions. Magn Reson Imaging 2006;24:1131-42

AJNR Am J Neuroradiol 35:439-44 Mar 2014 www.ajnr.org 
22. Kleihues P, Cavenee WB. World Health Organization Classification of Tumors: Pathology and Genetics: Tumors of the Nervous System. Lyon, France: IARC Press; 2000

23. Price SJ, Burnet NG, Donovan T, et al. Diffusion tensor imaging of brain tumours at $3 \mathrm{~T}$ : a potential tool for assessing white matter tract invasion? Clin Radiol 2003;58:455-62

24. Lu S, Ahn D, Johnson G, et al. Diffusion tensor MR imaging of intracranial neoplasia and associated peritumoral edema: introduction of the tumor infiltration index. Radiology 2004;232:221-28

25. Tropine A, Vucurevic G, Delani P, et al. Contribution of diffusion tensor imaging to delineation of gliomas and glioblastomas. J Magn Reson Imaging 2004;20:905-12

26. Provenzale JM, McGraw P, Mhatre P, et al. Peritumoral brain regions in gliomas and meningiomas: investigation with isotropic diffusion-weighted MR imaging and diffusion-tensor MR imaging. Radiology 2004;232:451-60

27. Deng Z, Yan Y, Zhong D, et al. Quantitative analysis of glioma cell invasion by diffusion tensor imaging. J Clin Neurosci 2010;17: $1530-06$

28. Wang W, Steward CE, Desmond PM. Diffusion tensor imaging in glioblastoma multiforme and brain metastases: the role of $\mathrm{p}, \mathrm{q}$, L, and fractional anisotropy. AJNR Am J Neuroradiol 2009; 30:203-08

29. Price SJ, Jena R, Burnet NG, et al. Improved delineation of glioma margins and regions of infiltration with the use of diffusion tensor imaging: an image-guided biopsy study. AJNR Am J Neuroradiol 2006;27:1969-74

30. Kinoshita M, Goto T, Okita $\mathrm{Y}$, et al. Diffusion tensor based tumor infiltration index cannot discriminate vasogenic edema from tumor infiltrated edema. J Neurooncol 2010;96:409-15

31. Chen W. Clinical applications of PET in brain tumors. J Nucl Med 2007;48:1468-81

32. Kato T, Shinoda J, Nakayama N, et al. Metabolic assessment of gliomas using $11 \mathrm{C}$-methionine, $[18 \mathrm{~F}]$ fluorodeoxyglucose, and $11 \mathrm{C}$ choline positron-emission tomography. AJNR Am J Neuroradiol 2008;29:1176-82

33. Kinoshita M, Hashimoto N, Goto T, et al. Use of fractional anisotropy for determination of the cut-off value in (11)C-methionine positron emission tomography for glioma. Neuroimage 2009;45: $312-18$ 